\title{
A Novel Solid State Pulsed Power Module for Excimer Laser
}

\author{
C. StrowitzKi*
}

FB 16, Elektrische Energieversorgungssysteme, Universität Kassel, Germany

\begin{abstract}
For effective excimer laser pumping high voltage pulses with the pulse duration of $100 \mathrm{~ns}$ and energies from a fraction of joules to some 10 joules are necessary. For the generation of these pulses traditionally Thyratron circuits are used. The limited lifetime and repetition rates of such a device does not fulfil not the requirements of a modern excimer laser. State of the art is a solid state circuit with IGBT switches, high voltage pulse transformer and pulse compression networks. Due to the many stages, complexity is high and efficiency is moderate. To overcome this limitation a new solid state circuit for excimer laser pumping is developed. The circuit works without magnetic pulse compression. The switch is made by a stack of "off the self" MOSFETs and SiC diodes. In the presentation the general design and its calculation, diode and switch qualification and test results are presented.
\end{abstract}

PACS numbers: $84.70 .+\mathrm{p}$

\section{Introduction}

Excimer lasers are gas discharge pumped lasers emitting light in the UV. Due to the short wavelength the pumping density in an excimer laser has to be very high. It is about $100 \mathrm{~J} / \mathrm{l}$. Due to the very short pumping time the pumping power density is remarkably high, about $10 \mathrm{GW} / \mathrm{l}$.

To provide such a high power density a capacitor discharge circuit is used [1]. The challenges of the laser head design are the low inductivity of the laser head $(20 \mathrm{nH}$ down to $1 \mathrm{nH}$ ), the gas tightness and the cleanness. The capacitors which feed the discharge are called peaking capacitors and normally located close to or even in the laser chamber.

The charging of these capacitors is the challenge of the pulsed power of excimer laser. The proposed circuit is especially well suited for small excimer laser with very high repetition rate (see bold letters).

TABLE

Typical requirements for the pulsed power of an excimer laser.

\begin{tabular}{|c|c|}
\hline Energy & $0.2 \mathbf{J}$ up to $50 \mathrm{~J}$ \\
\hline Voltage & $8 \mathbf{k V}$ up to $40 \mathrm{kV}$ \\
\hline Charge time & $50 \mathbf{n s}$ up to $150 \mathrm{~ns}$ \\
\hline Peaking capacity & $\mathbf{2} \mathbf{n F}$ up to $100 \mathrm{nF}$ \\
\hline Average power & up to $50 \mathrm{~kW}$ \\
\hline Repetition rate & up to $10 \mathrm{kHz}$ \\
\hline Laser light energy & $\mathbf{2} \mathbf{~ m J}$ up to $2 \mathrm{~J}$ \\
\hline
\end{tabular}

* e-mail: claus.strowitzki@coherent.com
The well known designs are the Thyratron circuit (with or without magnetic pulse compression) and the solid state circuit with magnetic pulse compression. In this work a new kind of circuit, a solid state circuit without magnetic pulse compression, is shown. The topology of the circuit is like a Thyratron circuit where the Thyratron is replaced by a stack of semiconductors.

\section{Circuit topology}

The topology of the circuit has to be chosen carefully:

- simple and straightforward,

- bank capacitors connected direct to ground (for fast charging),

- no high voltage pulse transformer for high efficiency,

- capable of handling the short circuit after the discharge.

The best design fulfilling these requirements is the $\mathrm{C}-\mathrm{C}$ transfer circuit (Fig. 1) The bank (C1) is grounded. As a consequence the switch (S1) floats. The laser discharge (CSx1) and the peakings (C2) are also related to ground.

After charging the bank to $15 \mathrm{kV}$ the switch is closed and the energy transfers in a resonant mode to the peaking capacitor. The peaking capacitors are charged to the voltage level where the discharge ignites and the peaking capacitors are depleted. The discharge acts then as a short circuit and the remaining energy of the bank and inductivity is reflected to charge the bank to the opposite of the origin voltage. To avoid a second turn the diode blocks and the energy is kept in the bank [2]. 

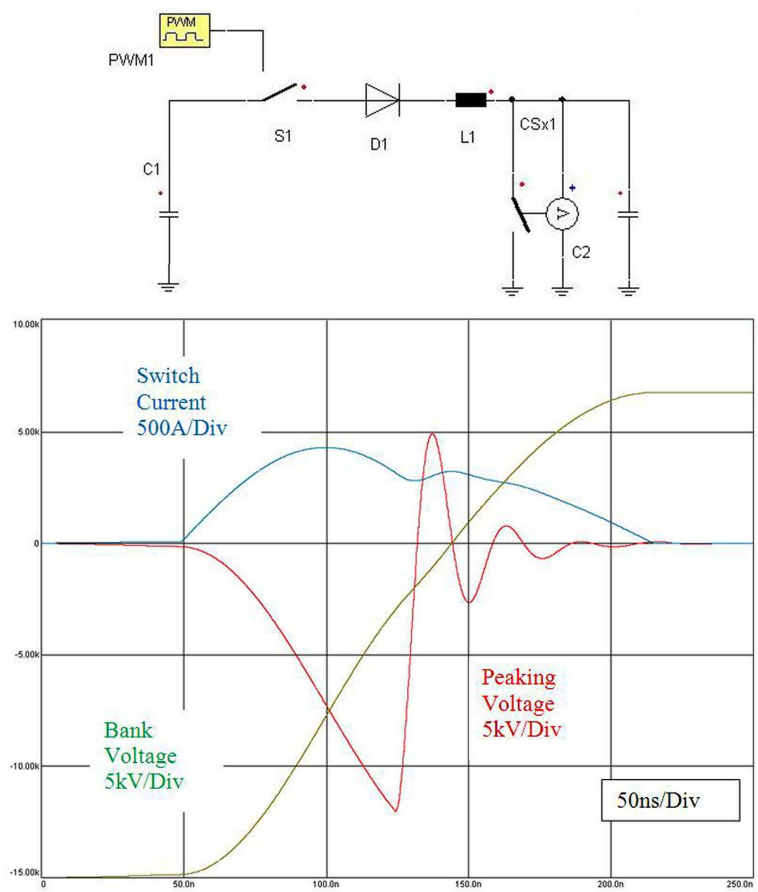

Fig. 1. Scheme of the circuit and simulation results. Switch current: 500 A/Div; Bank Voltage: $5 \mathrm{kV} /$ Div; Peaking Voltage $5 \mathrm{kV} /$ Div; Time scale: $40 \mathrm{~ns} /$ Div).

\section{Results and discussion}

The result of the simulation (Fig. 1) shows that in principle the circuit is feasible. The stress of the components of the circuit is very high but within the capabilities of modern semiconductors.

\section{Active components}

The very high speed of the circuit reduces the possible usable switch type and diode. IGBTs are not feasible because in this time frame switching losses are too high (in the range of $30 \%$ of the transferred energy).

In Fig. 2 the switching behaviour of IGBT and MOSFET is compared. The speed of the voltage drop is quite the same. The main difference is that during the current rise the voltage drop of the IGBT rises to unexpected values $(300 \mathrm{~V})$ and causes excessive losses. The voltage drop of the IGBT is only caused due to the "on resistance" and the inductivity (which do not contribute to the losses).

Different commercially available MOSFETs were tested. All of them behave more or less in the same way and show no big differences in performance.

Due to the speed of the circuit only Schottky diodes are feasible. For this high voltage condition only $\mathrm{SiC}$ diodes are available.

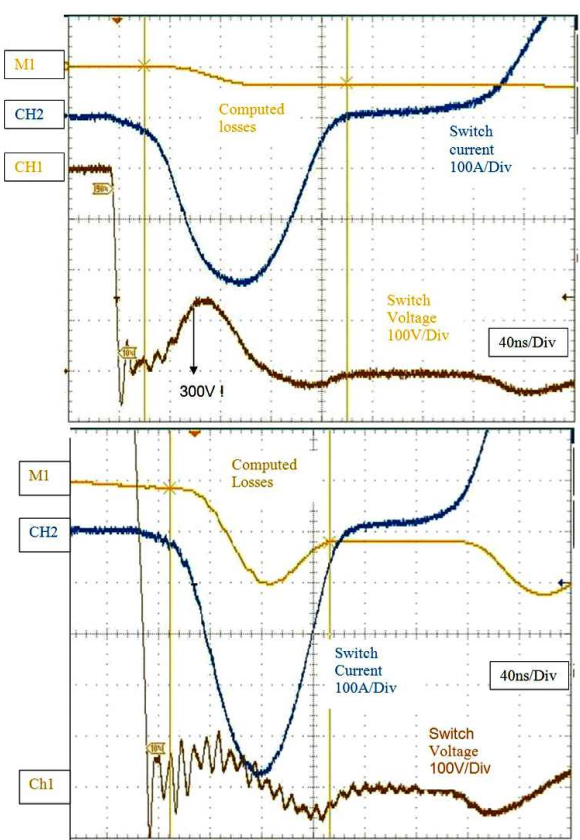

Fig. 2. Comparison of switching behaviour of IGBT (left) and MOSFET (right) (please note the different scaling). Switch voltage (CH1): $100 \mathrm{~V} /$ Div; Switch current (CH2) 100 A/Div; Computed losses (M1) arbitrary units; Time scale: $40 \mathrm{~ns} /$ Div.

\section{Voltage balancing}

To ensure a stable voltage and current share proper balancing is necessary. The current sharing in the circuit (four parallel components) is no problem. Due to the positive temperature coefficients of the used components proper current share is automatically achieved.

More caution has to be put into voltage sharing. Static voltage balancing is done with proper designed resistors (current is 10 times higher than the leakage current of the switch). For dynamic voltage sharing snupper like RC networks are possible. Also in this case schoolbook-like design (snupper capacity is ten times of the depletion layer capacity) works properly.

\section{Test results}

The circuit could be attached direct to the laser head. This is necessary because the overall inductivity of the circuit has to be about $1 \mu \mathrm{H}$.

The test result shows that the circuit works in the calculated way. The peaking capacitors are charged in less than $100 \mathrm{~ns}$. The energy which is reflected from the laser head is controlled properly.

A new resonant power supply was developed that allows charging times of less than $50 \mu$ s and operation of up to $6 \mathrm{kHz}$ is possible as shown in Fig. 3 . 


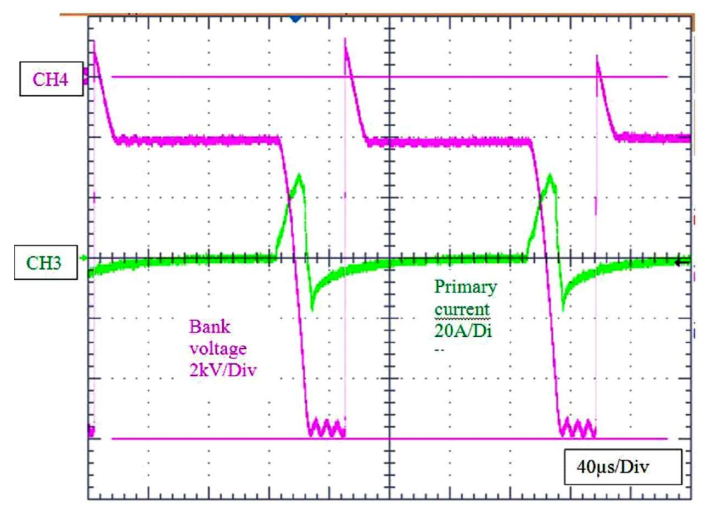

Fig. 3. Operation of the stack (CH3 charge current; $\mathrm{CH} 4$ Bank capacitor voltage) at $6 \mathrm{kHz}$. Charge current (CH3): $20 \mathrm{~A} /$ Div; Bank capacitor voltage (CH4): $2 \mathrm{kV} /$ Div; Time scale $40 \mu \mathrm{s} /$ Div.

\section{Conclusion}

A novel solid state modulator without pulse compression is presented. It shows that with off the shelf power semiconductors it is possible to pump a small very high repetition rate excimer laser.

\section{References}

[1] Magnetic Pulse Compression for Excimer Lasers, Questek, Technical Note, No. 1 and No. 2, 1983.

[2] C.F. Strowitzki, A novel solid state pulsed power module for excimer laser, Verhandlungen DPG (VI) 42,3/K9.2 (2007). 